\title{
Association of Short-Term Exposure to Meteorological Factors and Risk of Hand, Foot, and Mouth Disease: A Systematic Review and Meta-Analysis
}

\author{
Zhihui Liu ${ }^{1, \dagger}$, Yongna Meng ${ }^{1, \dagger}$, Hao Xiang ${ }^{1}{ }^{\mathbb{C}}$, Yuanan $\mathrm{Lu}^{2, *}$ and Suyang Liu ${ }^{1, *}$ \\ 1 Department of Global Health, School of Health Sciences, Wuhan University, Wuhan 430071, China; \\ zhizhiliu@whu.edu.cn (Z.L.); mengyongna@whu.edu.cn (Y.M.); xianghao@whu.edu.cn (H.X.) \\ 2 Environmental Health Laboratory, Department of Public Health Sciences, University of Hawaii at Manoa, \\ Honolulu, HI 96822, USA \\ * Correspondence: yuanan@hawaii.edu (Y.L.); dayangwater@hotmail.com (S.L.); Tel.: +86-027-68759118 (S.L.); \\ Fax: +86-027-68758648 (S.L.) \\ + The two authors made equal contributions to this article.
}

Received: 7 October 2020; Accepted: 27 October 2020; Published: 30 October 2020

\begin{abstract}
Background: Inconsistencies were observed in studies on the relationship between short-term exposure to meteorological factors and the risk of hand, foot, and mouth disease (HFMD). This systematic review and meta-analysis was aimed to assess the overall effects of meteorological factors on the incidence of HFMD to help clarify these inconsistencies and serve as a piece of evidence for policy makers to determine relevant risk factors. (2) Methods: Articles published as of 24 October 2020, were searched in the four databases, namely, PubMed, Web of Science, Embase, and MEDLINE. We applied a meta-analysis to assess the impact of ambient temperature, relative humidity, rainfall, wind speed, and sunshine duration on the incidence of HFMD. We conducted subgroup analyses by exposure metrics, exposure time resolution, regional climate, national income level, gender, and age as a way to seek the source of heterogeneity. (3) Results: Screening by the given inclusion and exclusion criteria, a total of 28 studies were included in the analysis. We observed that the incidence of HFMD based on the single-day lag model is significantly associated with ambient temperature, relative humidity, rainfall, and wind speed. In the cumulative lag model, ambient temperature and relative humidity significantly increased the incidence of HFMD as well. Subgroup analysis showed that extremely high temperature and relative humidity significantly increased the risk of HFMD. Temperate regions, high-income countries, and children under five years old are major risk factors for HFMD. (4) Conclusions: Our results suggest that various meteorological factors can increase the incidence of HFMD. Therefore, the general public, especially susceptible populations, should pay close attention to weather changes and take protective measures in advance.
\end{abstract}

Keywords: hand, foot, and mouth disease; infectious disease; meteorological factors; ambient temperature; relative humidity

\section{Introduction}

Hand, foot, and mouth disease (HFMD) is an airborne disease that causes a huge global disease burden [1]. HFMD was first discovered in New Zealand in 1957, and then gradually became known worldwide. In the past 20 years, HFMD outbreaks have become more and more common throughout the Asia-Pacific region [2,3]. Affected areas include, but are not limited to, Japan, South Korea, Singapore, Malaysia, Vietnam, and China. The main pathogens of HFMD are Coxsackie virus A16 (CA16) and Enterovirus 71 (EV71) [4]. Although HFMD is a self-limiting disease, its extensive harm to the human body cannot be ignored. The typical symptoms include fever, skin bursts on hands 
and feet, and vesicles in the mouth [2]. It is commonly seen in children between the ages of 0 and 15, especially children under five years of age [5]. Most HFMD patients suffer from complications such as myocarditis, pulmonary edema, aseptic meningoencephalitis, and even death. Therefore, identifying the environmental factors that are associated with HFMD has public health significance.

Over the past decade, the relationship between meteorological factors and HFMD has drawn widespread attention. However, the results are inconsistent, especially in terms of rainfall, wind speed, and sunshine duration. For example, Nguyen et al. (2017) [6] found that the increase in average weekly rainfall in nine provinces in the Mekong Delta region of Vietnam is associated with the increased risk of HFMD. However, Li et al. (2014) [7] observed no such association in Shanghai, China. Zhu et al. (2019) [8] found that the average daily sunshine duration is a protective factor for the risk of HFMD in Henan, China, while another similar Chinese study disapproved this observation in Shanghai, China [9]. Additionally, $\mathrm{Xu}$ et al. (2019) [10] found that the weekly minimum temperature was significantly associated with the increase the HFMD risk, while Hii et al. (2011) [11] found the weekly minimum temperature was a protective factor against HFMD. Yang et al. (2018) [12] and Hao et al. (2020) [13] observed that the daily average minimum relative humidity has an adverse effect on the risk of HFMD in Wuhan, China, while Nguyen et al. (2019) [14] found no such association in the Mekong Delta Region, Vietnam.

Due to inconsistent epidemiological studies mentioned above, we believe that a systematic review and meta-analysis may clarify these inconsistencies and help us see the big picture. Prior to this work, we were fully aware that a meta-analysis published in 2018 has done similar work [15]. However, we still decided to write this article for the following reasons: (1) After the publication of this study, a large number of epidemiological studies on HFMD and meteorological factors have been published in the scientific community, and the total number has doubled. (2) In the previous study, only two meteorological factors, ambient temperature and relative humidity, were included. In our study, rainfall, wind speed, and sunshine duration were also added into the analysis. (3) Our study not only includes effect estimates generated from single-day lag models, but also those from cumulative-day lag models. (4) In addition, we are interested in knowing whether the relationship between ambient temperature and relative humidity and HFMD risk has changed over time by comparing our results with ones reported by the previous meta-analysis.

With these concerns in mind, we conducted this study to systematically assess the impact of meteorological factors on the risk of HFMD, hoping to help policy makers identify high-risk factors and protect susceptible populations.

\section{Methods}

\subsection{Search Strategy}

To find the related articles, we searched databases including PubMed, Web of Science, Embase, and MEDLINE using the following keywords: "'hand, foot and mouth disease" or "hand foot mouth disease" or "hand-foot-mouth disease" or "HFMD") AND ("ambient temperature" or "temperature" or "humidity" or "relative humidity" or "climate" or "meteorology" or "weather" or "rainfall" or "precipitation" or "atmospheric pressure" or "barometric pressure "or "air pressure" or "wind speed" or "wind velocity" or "sunshine" or "sunshine duration"). Studies published up to the date of the search (24 October 2020) were considered.

\subsection{Eligibility and Selection Criteria}

The inclusion criteria of our literature were as follows: (1) studies were written in English, or in other languages if an English translation was available; (2) studies provided effect estimates of relative risk (RR), incidence rate ratio (IRR), or the excess risk (ER); (3) studies reported cases of HFMD rather than death; (4) the associations were reported as effect estimates calculated based on one unit increase in meteorological factors (e.g., RR and 95\% confidence intervals [Cls] of HFMD associated with each 
$1{ }^{\circ} \mathrm{C}$ increase in ambient temperature. Additionally, RRs that can be converted to standardized RR were also included $[15,16]$.

The exclusion criteria of our literature were as follows: (1) the full text of the article could not be found; (2) the format of the paper is not a research article; (3) articles do not contain data for meta-analysis; and (4) articles do not estimate the effect of confounding from any source.

\subsection{Data Extraction}

The titles and abstracts derived from the databases have been downloaded to the reference manager Endnote (Version X8, developed by Clarivate Analytics, Philadelphia, United States) prior to the analysis. All records were independently evaluated by two investigators, and disputes are arbitrated by the third investigator. Upon removing the irrelevant articles and duplicate studies, the articles that met the selection criteria were included in our analysis. We extracted the following information from eligible articles: (1) study characteristics, (2) participants' demographic factors, (3) the relative risks and 95\% CLs of HFMD associated with meteorological factors, (4) outcomes of studies.

\subsection{Quality Assessment}

The quality of the selected articles was assessed by a Newcastle Ottawa Scale (NOS). The NOS included eight items in three dimensions [17]. Two investigators in our lab independently assessed the quality of the items according to the NOS guidelines, and the third arbitrator resolved the disagreement between two investigators if there was any. The results are shown in Supplementary Material Table S1. We only included articles with a NOS score of 6-9 to ensure that all studies included were of moderate or high quality.

\subsection{The Selection of Lag Effects and Exposure Metrics}

In selected studies, reporting associations on different lag days is a common practice. Among them, most studies were interested in single-day lag effects [6,11,18-25], while others focused on cumulative lag effects [12,26-29]. In our study, we selected lag days for analysis according to the criteria proposed by Atkinson et al. (2012) [30] and Yang et al. (2018) [31]. In short, if the results of an article were based on a single-day lag, then its results were included in the analysis completely. If an article was based on multiple lag days, we selected effects to be included in the analysis according to the following criteria: (1) the authors clearly indicated the priority of a certain lag, (2) the results were statistically significant on this lag, and (3) the lag days on which the associations of HFMD with both the maximum and minimum exposures were reported.

As for the exposure metrics, we selected results according to the following criteria. If only one exposure metric was presented (typically it is the mean exposure), then the effect of these metrics was included in the analysis. If associations of HFMD with the maximum and minimum exposure were reported rather than the mean exposure, then both were included. Additionally, if a study included results from more than one location, then results from all locations were included.

\subsection{Statistical Analysis}

Forest plots were used to assess the heterogeneity of the associations of HFMD with temperature, relative humidity, wind speed, rainfall, and sunshine duration. Both the Cochran $Q$ [32] and the I-squared $\left(\mathrm{I}^{2}\right)$ statistics [33] were used to assess the heterogeneity. We conducted several subgroup analyses to explore the sources of heterogeneity, namely, gender (females vs. males), age ( $\leq 5$ years vs. $>5$ years), exposure metrics (maximum vs. mean vs. minimum), exposed time resolution (daily vs. weekly vs. monthly), regional climate (tropical vs. temperate), and human development index (HDI) rank, a proxy for a country's national income (high vs. low). The classification of HDI was based on the United Nations definition of low- and high-income countries [34]. To report the results, we mainly used RRs as the effect estimates. Two of the selected articles reported ER, and one reported IRR. We regarded them as RR, as they were approximately equal to RR [19]. 
Stata (version 12.0, Stata Corp. LLC, College Station, TX, USA) was used for data cleaning and merging. The "forest" package in R software (version 3.5.3, R Foundation for Statistical Computing, Vienna, Austria) was used to perform the meta-analysis. Publication bias was assessed by the Begg rank correlation test and Egger weighted regression test [35,36].

To assess the stability of the aggregated estimates, two sensitivity analyses were performed. The first sensitivity analysis was conducted to compare whether there is a difference in associations between meteorological factors and HFMD with extreme exposure values included or not, while the second sensitivity analysis was to compare the differences in effects, which has been previously reported by Cheng et al. (2018) [15] and effects generated by later updated literatures.

\section{Results}

\subsection{Study Selection and Characteristics}

A total of 10,668 records were searched through the first round of screening. After removing articles that did not meet the inclusion criteria, 28 studies were kept in this study. The screening process is presented in Figure 1. The characteristics of the selected articles were shown in Table 1. Among them, 25 studies assessed the associations of HFMD with ambient temperature, 23 with relative humidity, 11 with rainfall, 7 with wind speed, and 5 with sunshine duration for those focused on the single-day lag effect. Meanwhile, 14 studies assessed the associations of HFMD with ambient temperature and 10 studies with relative humidity based on cumulative lag models. The most commonly used statistical model is the generalized additive model (GAM). Case-crossover models and negative binomial regression are also used. Some articles used the distributed lag non-linear model (DLNM) and the above models together to assess the combined effect of time lags and meteorological factors on HFMD. All articles were conducted in Asia-Pacific regions. The majority of the selected articles were conducted in China, with the rest carried out in Japan, Korea, Singapore, and Vietnam.

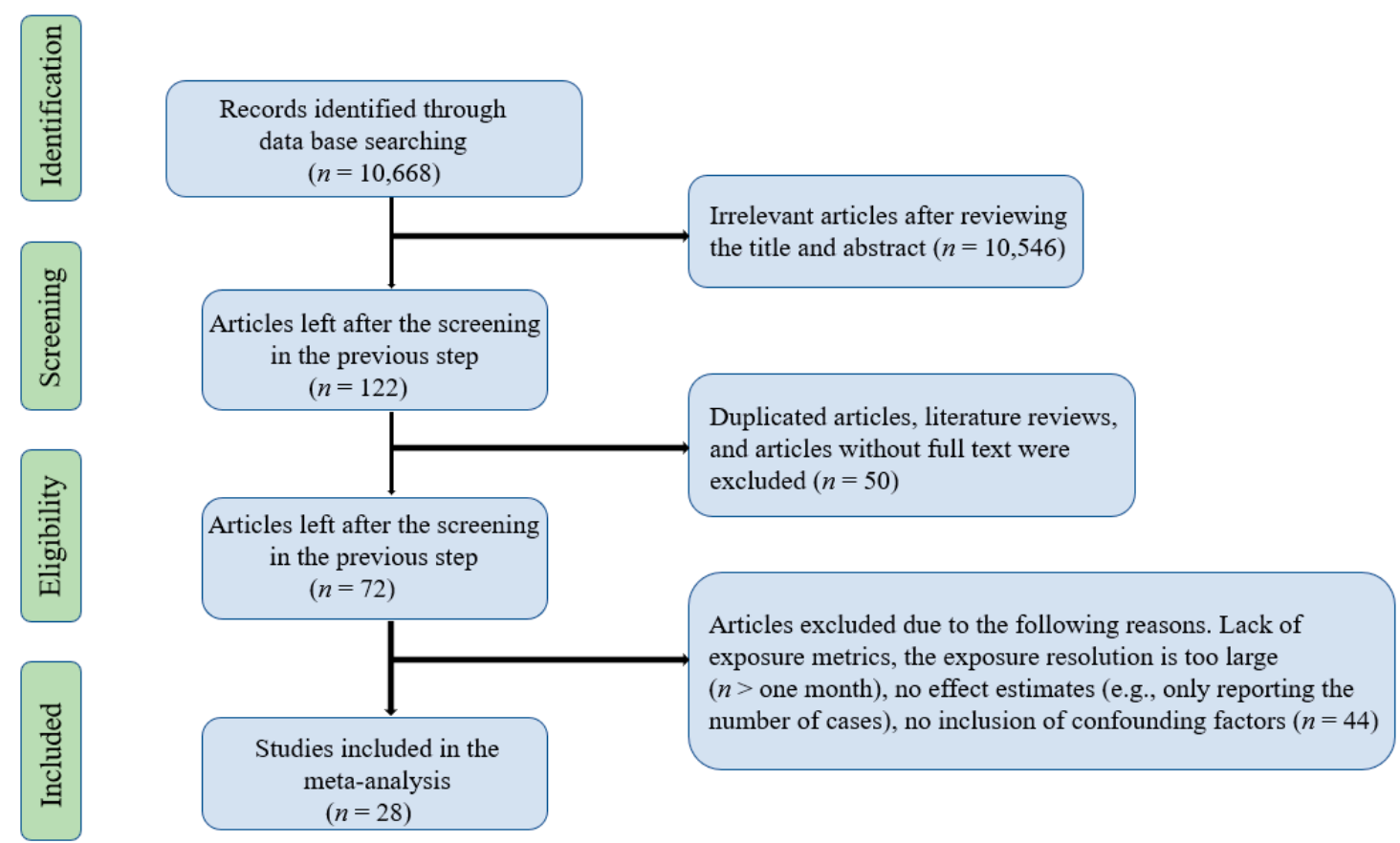

Figure 1. Flowchart showing the screening process for included articles. 
Table 1. Characteristics of the included studies on the associations between meteorological factors and the risk of HFMD.

\begin{tabular}{|c|c|c|c|c|c|c|c|c|c|c|c|c|c|}
\hline Reference & $\begin{array}{l}\text { Study } \\
\text { Location }\end{array}$ & $\begin{array}{l}\text { Study } \\
\text { Period }\end{array}$ & Population & Ages & Exposure Variable & Statistical Model & $\begin{array}{l}\text { Temporal } \\
\text { Lags }\end{array}$ & Resolution & $\begin{array}{l}\text { Climate } \\
\text { Group }\end{array}$ & $\begin{array}{l}\text { Measure } \\
\text { Index }\end{array}$ & $\begin{array}{l}\text { HDI } \\
\text { Rank }\end{array}$ & $\begin{array}{l}\text { Quality } \\
\text { Scores }\end{array}$ & Outcome \\
\hline $\begin{array}{l}\text { Zhu et al. } \\
\text { (2015) [29] }\end{array}$ & $\begin{array}{l}\text { Shandong, } \\
\text { China }\end{array}$ & 2007-2012 & 108,377 & $\begin{array}{l}0-5 \\
\text { years }\end{array}$ & $\begin{array}{l}\text { Cumulative maximum } \\
\text { temperature; cumulative } \\
\text { minimum temperature }\end{array}$ & $\begin{array}{l}\text { Distributed lag } \\
\text { non-linear model } \\
\text { (DLNM) with Poisson } \\
\text { distribution, adjusting } \\
\text { for relative humidity, } \\
\text { rainfall, sunshine } \\
\text { duration, DOW, public } \\
\text { holidays, seasonal trend, } \\
\text { and long trend }\end{array}$ & $\begin{array}{l}0-14 \\
\text { days }\end{array}$ & Daily & $\begin{array}{l}\text { Temperate } \\
\text { climate }\end{array}$ & $\mathrm{RR}$ & High & 8 & $\begin{array}{l}\text { Reported } \\
\text { HFMD }\end{array}$ \\
\hline $\begin{array}{l}\text { Chen et al. } \\
\text { (2014) [19] }\end{array}$ & $\begin{array}{l}\text { Guangzhou, } \\
\text { China }\end{array}$ & 2009-2011 & 34,527 & $\begin{array}{l}0-14 \\
\text { years }\end{array}$ & $\begin{array}{l}\text { Mean temperature; relative } \\
\text { humidity }\end{array}$ & $\begin{array}{l}\text { Generalized additive } \\
\text { model (GAM), } \\
\text { adjusting for long-term } \\
\text { trend, seasonal trend, } \\
\text { day of week, and public } \\
\text { holidays }\end{array}$ & $\begin{array}{l}0-10 \\
\text { days }\end{array}$ & Daily & $\begin{array}{l}\text { Temperate } \\
\text { climate }\end{array}$ & IRR & High & 7 & $\begin{array}{l}\text { Reported } \\
\text { HFMD }\end{array}$ \\
\hline $\begin{array}{l}\text { Yang et al. } \\
\text { (2018) [12] }\end{array}$ & Hefei, China & 2011-2016 & NA & All & $\begin{array}{l}\text { Mean temperature; rainfall; } \\
\text { cumulative mean relative } \\
\text { humidity }\end{array}$ & $\begin{array}{l}\text { DLNM, adjusting for } \\
\text { long-term trend, } \\
\text { seasonal trend, and day } \\
\text { of week }\end{array}$ & $\begin{array}{l}0-20 \\
\text { days }\end{array}$ & Daily & $\begin{array}{l}\text { Temperate } \\
\text { climate }\end{array}$ & $R R$ & High & 8 & $\begin{array}{l}\text { Reported } \\
\text { HFMD }\end{array}$ \\
\hline $\begin{array}{l}\text { Xu et al. } \\
\text { (2015) [37] }\end{array}$ & $\begin{array}{l}\text { Beijing, } \\
\text { China }\end{array}$ & 2010-2012 & 113,475 & $\begin{array}{l}6-15 \\
\text { years }\end{array}$ & $\begin{array}{l}\text { Mean temperature; relative } \\
\text { humidity; cumulative } \\
\text { maximum temperature; } \\
\text { cumulative minimum } \\
\text { temperature }\end{array}$ & $\begin{array}{l}\text { A newly developed } \\
\text { case-crossover design } \\
\text { with DLNM, adjusting } \\
\text { for day of week, public } \\
\text { holidays, long-term } \\
\text { trend, and seasonal } \\
\text { trend }\end{array}$ & $\begin{array}{l}0-13 \\
\text { days }\end{array}$ & Daily & $\begin{array}{l}\text { Temperate } \\
\text { climate }\end{array}$ & RRs & High & 7 & $\begin{array}{l}\text { Reported } \\
\text { HFMD }\end{array}$ \\
\hline $\begin{array}{l}\text { Yu et al. } \\
\text { (2018) [28] }\end{array}$ & $\begin{array}{l}\text { Guilin, } \\
\text { China }\end{array}$ & 2014-2016 & 88,742 & $\begin{array}{l}0-14 \\
\text { years }\end{array}$ & $\begin{array}{l}\text { Relative humidity; sunshine } \\
\text { duration; wind speed; rainfall; } \\
\text { cumulative maximum } \\
\text { temperature; cumulative } \\
\text { minimum temperature; } \\
\text { cumulative maximum } \\
\text { relative humidity; cumulative } \\
\text { minimum relative humidity; }\end{array}$ & $\begin{array}{l}\text { DLNM, adjusting for } \\
\text { long-term trends, } \\
\text { seasonality, differences } \\
\text { in the annual at-risk } \\
\text { population; day of week, } \\
\text { and public holidays }\end{array}$ & $\begin{array}{l}0-14 \\
\text { days }\end{array}$ & Daily & $\begin{array}{l}\text { Temperate } \\
\text { climate }\end{array}$ & $\mathrm{RR}$ & High & 7 & $\begin{array}{l}\text { Reported } \\
\text { HFMD }\end{array}$ \\
\hline $\begin{array}{l}\text { Zhang et al. } \\
\text { (2018) [38] }\end{array}$ & $\begin{array}{l}\text { Henan, } \\
\text { China }\end{array}$ & 2012-2013 & NA & $\begin{array}{l}0-5 \\
\text { years }\end{array}$ & $\begin{array}{l}\text { Mean temperature; relative } \\
\text { humidity; rainfall; sunshine } \\
\text { duration; wind speed }\end{array}$ & $\begin{array}{l}\text { Bayesian space-time } \\
\text { hierarchy mode, } \\
\text { Poisson with log link } \\
\text { regression and } \\
\text { GeoDetector, adjusting } \\
\text { for long-term trend and } \\
\text { autocorrelation }\end{array}$ & None & None & $\begin{array}{l}\text { Temperate } \\
\text { climate }\end{array}$ & $\mathrm{RR}$ & High & 6 & $\begin{array}{l}\text { Reported } \\
\text { HFMD }\end{array}$ \\
\hline
\end{tabular}


Table 1. Cont.

\begin{tabular}{|c|c|c|c|c|c|c|c|c|c|c|c|c|c|}
\hline Reference & $\begin{array}{l}\text { Study } \\
\text { Location }\end{array}$ & $\begin{array}{l}\text { Study } \\
\text { Period }\end{array}$ & Population & Ages & Exposure Variable & Statistical Model & $\begin{array}{l}\text { Temporal } \\
\text { Lags }\end{array}$ & Resolution & $\begin{array}{l}\text { Climate } \\
\text { Group }\end{array}$ & $\begin{array}{l}\text { Measure } \\
\text { Index }\end{array}$ & $\begin{array}{l}\text { HDI } \\
\text { Rank }\end{array}$ & $\begin{array}{l}\text { Quality } \\
\text { Scores }\end{array}$ & Outcome \\
\hline $\begin{array}{l}\text { Qi et al. } \\
\text { (2018) [9] }\end{array}$ & $\begin{array}{l}\text { Shanghai, } \\
\text { China }\end{array}$ & 2009-2015 & 51,776 & $\begin{array}{l}0-15 \\
\text { years }\end{array}$ & $\begin{array}{l}\text { Mean temperature; relative } \\
\text { humidity }\end{array}$ & $\begin{array}{l}\text { DLNM, adjusting for } \\
\text { potential confounders of } \\
\text { long time trend, DOW, } \\
\text { and public holidays }\end{array}$ & $\begin{array}{l}0-14 \\
\text { days }\end{array}$ & Daily & $\begin{array}{l}\text { Temperate } \\
\text { climate }\end{array}$ & $\mathrm{RR}$ & High & 8 & $\begin{array}{l}\text { Reported } \\
\text { HFMD }\end{array}$ \\
\hline $\begin{array}{l}\text { Zhu et al. } \\
\text { (2016) [21] }\end{array}$ & $\begin{array}{l}\text { Shandong, } \\
\text { China }\end{array}$ & 2007-2012 & 504,017 & $\begin{array}{l}0-5 \\
\text { years }\end{array}$ & Mean temperature & $\begin{array}{l}\text { DLNM, adjusting for } \\
\text { seasonal trend, long } \\
\text { time trend, DOW, and } \\
\text { public holidays }\end{array}$ & $\begin{array}{l}0-21 \\
\text { days }\end{array}$ & Daily & $\begin{array}{l}\text { Temperate } \\
\text { climate }\end{array}$ & RR & High & 9 & $\begin{array}{l}\text { Reported } \\
\text { HFMD }\end{array}$ \\
\hline $\begin{array}{l}\text { Bo et al. } \\
\text { (2020) [22] }\end{array}$ & $\begin{array}{l}\text { 143city, } \\
\text { China }\end{array}$ & 2009-2014 & $3,060,450$ & $\begin{array}{l}0-12 \\
\text { years }\end{array}$ & Relative humidity & $\begin{array}{l}\text { DLNM, adjusting for } \\
\text { long-term trends, } \\
\text { seasonality, } \\
\text { autocorrelation, DOW } \\
\text { public holidays }\end{array}$ & $\begin{array}{l}0-18 \\
\text { days }\end{array}$ & Daily & None & $\mathrm{RR}$ & High & 9 & $\begin{array}{l}\text { Reported } \\
\text { HFMD }\end{array}$ \\
\hline $\begin{array}{l}\text { Wang et al. } \\
\text { (2016) [39] }\end{array}$ & $\begin{array}{l}\text { Hong Kong, } \\
\text { China }\end{array}$ & 2009-2014 & 1534 & All & $\begin{array}{l}\text { Rainfall; wind speed; } \\
\text { sunshine duration; } \\
\text { cumulative mean } \\
\text { temperature: cumulative } \\
\text { maximum relative } \\
\text { cumulative minimum relative } \\
\text { humidity }\end{array}$ & $\begin{array}{l}\text { A combination of } \\
\text { negative binomial } \\
\text { generalized additive } \\
\text { models and DLNM, } \\
\text { adjusting for multiple } \\
\text { environmental factors, } \\
\text { long-term trends, and } \\
\text { seasonality }\end{array}$ & $\begin{array}{l}0-30 \\
\text { days }\end{array}$ & Daily & $\begin{array}{l}\text { Tropical } \\
\text { climate }\end{array}$ & RR & High & 7 & $\begin{array}{l}\text { Reported } \\
\text { HFMD }\end{array}$ \\
\hline $\begin{array}{l}\text { Yin et al. } \\
\text { (2016) [18] }\end{array}$ & $\begin{array}{l}\text { Chengdu, } \\
\text { China }\end{array}$ & 2010-2013 & 76,403 & $\begin{array}{l}0-14 \\
\text { years }\end{array}$ & Mean temperature & $\begin{array}{l}\text { DLNM, adjusting for } \\
\text { seasonal trend, long } \\
\text { time trend, DOW, and } \\
\text { holidays }\end{array}$ & $\begin{array}{l}0-13 \\
\text { days }\end{array}$ & Daily & $\begin{array}{l}\text { Temperate } \\
\text { climate }\end{array}$ & $\mathrm{RR}$ & High & 7 & $\begin{array}{l}\text { Reported } \\
\text { HFMD }\end{array}$ \\
\hline $\begin{array}{l}\text { Guo et al. } \\
\text { (2016) [26] }\end{array}$ & $\begin{array}{l}\text { Guangdong, } \\
\text { China }\end{array}$ & 2009-2013 & 400,408 & $\begin{array}{l}0-14 \\
\text { years }\end{array}$ & $\begin{array}{l}\text { Relative humidity; } \\
\text { cumulative mean } \\
\text { temperature; cumulative } \\
\text { mean relative humidity }\end{array}$ & $\begin{array}{l}\text { A mixed generalized } \\
\text { additive models } \\
\text { (MGAM), adjusting for } \\
\text { seasonal trend, long } \\
\text { time trend, DOW, and } \\
\text { holidays }\end{array}$ & $\begin{array}{l}0-14 \\
\text { days }\end{array}$ & Daily & $\begin{array}{l}\text { Temperate } \\
\text { climate }\end{array}$ & $\mathrm{RR}$ & High & 7 & $\begin{array}{l}\text { Reported } \\
\text { HFMD }\end{array}$ \\
\hline $\begin{array}{l}\text { Hao et al. } \\
\text { (2020) [13] }\end{array}$ & $\begin{array}{l}\text { Wuhan, } \\
\text { China }\end{array}$ & 2013-2017 & NA & All & $\begin{array}{l}\text { Mean temperature; } \\
\text { cumulative maximum } \\
\text { temperature; cumulative } \\
\text { minimum temperature; } \\
\text { relative humidity; cumulative } \\
\text { maximum relative humidity; } \\
\text { cumulative minimum relative } \\
\text { humidity }\end{array}$ & $\begin{array}{l}\text { DLNM combined with } \\
\text { Poisson regression, } \\
\text { adjusting for DOW, } \\
\text { seasonality, and } \\
\text { long-term time trend }\end{array}$ & $\begin{array}{l}0-14 \\
\text { days }\end{array}$ & Daily & $\begin{array}{l}\text { Temperate } \\
\text { climate }\end{array}$ & RR & High & 7 & $\begin{array}{l}\text { Reported } \\
\text { HFMD }\end{array}$ \\
\hline
\end{tabular}


Table 1. Cont.

\begin{tabular}{|c|c|c|c|c|c|c|c|c|c|c|c|c|c|}
\hline Reference & $\begin{array}{l}\text { Study } \\
\text { Location }\end{array}$ & $\begin{array}{l}\text { Study } \\
\text { Period }\end{array}$ & Population & Ages & Exposure Variable & Statistical Model & $\begin{array}{l}\text { Temporal } \\
\text { Lags }\end{array}$ & Resolution & $\begin{array}{l}\text { Climate } \\
\text { Group }\end{array}$ & $\begin{array}{l}\text { Measure } \\
\text { Index }\end{array}$ & $\begin{array}{l}\text { HDI } \\
\text { Rank }\end{array}$ & $\begin{array}{l}\text { Quality } \\
\text { Scores }\end{array}$ & Outcome \\
\hline $\begin{array}{l}\text { Xuan et al. } \\
\text { (2017) [6] }\end{array}$ & $\begin{array}{l}\text { Can Tho, } \\
\text { Vietnam }\end{array}$ & 2012-2014 & NA & All & $\begin{array}{l}\text { Mean temperature; relative } \\
\text { humidity }\end{array}$ & $\begin{array}{l}\text { Time-series regression } \\
\text { analysis, adjusting for } \\
\text { seasonality, long-term } \\
\text { time trend, DOW, and } \\
\text { the offset of population }\end{array}$ & $0-6$ days & Daily & $\begin{array}{l}\text { Tropical } \\
\text { climate }\end{array}$ & ER & Low & 7 & $\begin{array}{l}\text { Reported } \\
\text { HFMD }\end{array}$ \\
\hline $\begin{array}{l}\text { Gou et al. } \\
\text { (2018) [23] }\end{array}$ & $\begin{array}{l}\text { Gansu, } \\
\text { China }\end{array}$ & 2010-2014 & NA & All & $\begin{array}{l}\text { Mean temperature; relative } \\
\text { humidity }\end{array}$ & $\begin{array}{l}\text { Generalized linear } \\
\text { regression models } \\
\text { (GLM) with Poisson link } \\
\text { and classification and } \\
\text { regression trees (CART), } \\
\text { adjusting for seasonality }\end{array}$ & $\begin{array}{l}0-12 \\
\text { weeks }\end{array}$ & Weekly & $\begin{array}{l}\text { Temperate } \\
\text { climate }\end{array}$ & ER & High & 6 & $\begin{array}{l}\text { Reported } \\
\text { HFMD }\end{array}$ \\
\hline $\begin{array}{l}\text { Onozuka } \\
\text { et al. (2011) } \\
\text { [24] }\end{array}$ & $\begin{array}{l}\text { Fukuoka, } \\
\text { Japan }\end{array}$ & $2000-2010$ & 73,684 & All & $\begin{array}{l}\text { Mean temperature; relative } \\
\text { humidity }\end{array}$ & $\begin{array}{l}\text { Negative binomial } \\
\text { regression, adjusting for } \\
\text { seasonal and } \\
\text { inter-annual variations }\end{array}$ & $\begin{array}{l}0-3 \\
\text { weeks }\end{array}$ & Weekly & $\begin{array}{l}\text { Temperate } \\
\text { climate }\end{array}$ & $\mathrm{RR}$ & High & 7 & $\begin{array}{l}\text { Reported } \\
\text { HFMD }\end{array}$ \\
\hline $\begin{array}{l}\text { Hii et al. } \\
\text { (2011) [11] }\end{array}$ & Singapore & 2001-2008 & NA & All & $\begin{array}{l}\text { Maximum temperature } \\
\text { minimum temperature }\end{array}$ & $\begin{array}{l}\text { Time series Poisson } \\
\text { regression models, } \\
\text { adjusting for seasonality, } \\
\text { long-term time trend, } \\
\text { and autocorrelation }\end{array}$ & $\begin{array}{l}0-2 \\
\text { weeks }\end{array}$ & Weekly & $\begin{array}{l}\text { Temperate } \\
\text { climate }\end{array}$ & $\mathrm{RR}$ & High & 8 & $\begin{array}{l}\text { Reported } \\
\text { HFMD }\end{array}$ \\
\hline $\begin{array}{l}\text { Tian et al. } \\
\text { (2018) [40] }\end{array}$ & $\begin{array}{l}\text { Beijing, } \\
\text { China }\end{array}$ & 2010-2012 & 114,777 & $\begin{array}{l}0-4 \\
\text { years }\end{array}$ & $\begin{array}{l}\text { Mean temperature; relative } \\
\text { humidity; wind speed; } \\
\text { sunshine duration }\end{array}$ & $\begin{array}{l}\text { Bayesian spatiotemporal } \\
\text { Poisson regression } \\
\text { models; adjusting for } \\
\text { seasonality and } \\
\text { inter-annual variations }\end{array}$ & None & None & $\begin{array}{l}\text { Temperate } \\
\text { climate }\end{array}$ & $\mathrm{RR}$ & High & 7 & $\begin{array}{l}\text { Reported } \\
\text { HFMD }\end{array}$ \\
\hline $\begin{array}{l}\text { Kim et al. } \\
\text { (2016) [25] }\end{array}$ & South Korea & 2010-2013 & 214,642 & All & $\begin{array}{l}\text { Mean temperature; relative } \\
\text { humidity }\end{array}$ & $\begin{array}{l}\text { GAM and Poisson } \\
\text { distribution, controlling } \\
\text { for seasonality, } \\
\text { long-term time trend, } \\
\text { and autocorrelation }\end{array}$ & $\begin{array}{l}0-2 \\
\text { weeks }\end{array}$ & Weekly & $\begin{array}{l}\text { Temperate } \\
\text { climate }\end{array}$ & $\mathrm{RR}$ & High & 8 & $\begin{array}{l}\text { Reported } \\
\text { HFMD }\end{array}$ \\
\hline $\begin{array}{l}\text { Xuan et al. } \\
\text { (2019) [14] }\end{array}$ & $\begin{array}{l}\text { Mekong } \\
\text { Delta region, } \\
\text { Vietnam }\end{array}$ & $2014-2016$ & NA & $\begin{array}{l}0-5 \\
\text { years }\end{array}$ & $\begin{array}{l}\text { Mean temperature; humidity; } \\
\text { cumulative rainfall }\end{array}$ & $\begin{array}{l}\text { DLNM with } \\
\text { quasi-Poisson, } \\
\text { controlling for } \\
\text { long-term trend and } \\
\text { autocorrelation }\end{array}$ & $\begin{array}{l}0-20 \\
\text { days }\end{array}$ & Daily & $\begin{array}{l}\text { Temperate } \\
\text { climate }\end{array}$ & $\mathrm{RR}$ & High & 7 & $\begin{array}{l}\text { Reported } \\
\text { HFMD }\end{array}$ \\
\hline $\begin{array}{l}\text { Li et al. } \\
\text { (2014) [7] }\end{array}$ & $\begin{array}{l}\text { Guangzhou, } \\
\text { China }\end{array}$ & 2009-2012 & 166,770 & All & $\begin{array}{l}\text { Mean temperature; relative } \\
\text { humidity }\end{array}$ & $\begin{array}{l}\text { Negative binomial } \\
\text { multivariable } \\
\text { regression, adjusting for } \\
\text { long-term trend and } \\
\text { autocorrelation }\end{array}$ & None & Weekly & $\begin{array}{l}\text { Temperate } \\
\text { climate }\end{array}$ & ER & High & 6 & $\begin{array}{l}\text { Reported } \\
\text { HFMD }\end{array}$ \\
\hline
\end{tabular}


Table 1. Cont.

\begin{tabular}{|c|c|c|c|c|c|c|c|c|c|c|c|c|c|}
\hline Reference & $\begin{array}{l}\text { Study } \\
\text { Location }\end{array}$ & $\begin{array}{l}\text { Study } \\
\text { Period }\end{array}$ & Population & Ages & Exposure Variable & Statistical Model & $\begin{array}{l}\text { Temporal } \\
\text { Lags }\end{array}$ & Resolution & $\begin{array}{l}\text { Climate } \\
\text { Group }\end{array}$ & $\begin{array}{l}\text { Measure } \\
\text { Index }\end{array}$ & $\begin{array}{l}\text { HDI } \\
\text { Rank }\end{array}$ & $\begin{array}{l}\text { Quality } \\
\text { Scores }\end{array}$ & Outcome \\
\hline $\begin{array}{l}\text { Xu et al. } \\
\text { (2019) [10] }\end{array}$ & $\begin{array}{l}\text { Guangdong, } \\
\text { China }\end{array}$ & 2010-2013 & $1,048,574$ & $\begin{array}{l}0-5 \\
\text { years }\end{array}$ & $\begin{array}{l}\text { Mean temperature; maximum } \\
\text { temperature; minimum } \\
\text { temperature; mean relative } \\
\text { humidity; mean wind speed; } \\
\text { rainfall; sunshine duration; } \\
\text { cumulative maximum } \\
\text { temperature; cumulative } \\
\text { minimum temperature; } \\
\text { cumulative mean } \\
\text { temperature }\end{array}$ & $\begin{array}{l}\text { DLNM with } \\
\text { quasi-Poisson, } \\
\text { controlling for } \\
\text { long-term trend and } \\
\text { autocorrelation }\end{array}$ & $\begin{array}{l}0-21 \\
\text { days }\end{array}$ & Daily & $\begin{array}{l}\text { Temperate } \\
\text { climate }\end{array}$ & $\mathrm{RR}$ & High & 7 & $\begin{array}{l}\text { Reported } \\
\text { HFMD }\end{array}$ \\
\hline $\begin{array}{l}\text { Yang et al. } \\
\text { (2015) [41] }\end{array}$ & Hefei, China & 2010-2012 & 21,634 & $\begin{array}{l}0-14 \\
\text { years }\end{array}$ & Relative humidity & $\begin{array}{l}\text { Poisson linear } \\
\text { regression model and } \\
\text { DLNM, adjusting for } \\
\text { mean temperature, } \\
\text { seasonal patterns, and } \\
\text { long-term trends, day of } \\
\text { week }\end{array}$ & $\begin{array}{l}0-21 \\
\text { days }\end{array}$ & Daily & $\begin{array}{l}\text { Temperate } \\
\text { climate }\end{array}$ & ER & High & 7 & $\begin{array}{l}\text { Reported } \\
\text { HFMD }\end{array}$ \\
\hline $\begin{array}{l}\text { Zhu et al. } \\
\text { (2019) [8] }\end{array}$ & $\begin{array}{l}\text { Xiamen, } \\
\text { China }\end{array}$ & 2013-2017 & 36,464 & All & $\begin{array}{l}\text { Mean temperature; relative } \\
\text { humidity; sunshine duration }\end{array}$ & $\begin{array}{l}\text { DLNM with } \\
\text { quasi-Poisson, adjusting } \\
\text { for long-term time } \\
\text { trend, DOW, } \\
\text { and public holidays }\end{array}$ & $\begin{array}{l}0-20 \\
\text { days }\end{array}$ & Daily & $\begin{array}{l}\text { Temperate } \\
\text { climate }\end{array}$ & $\mathrm{RR}$ & High & 7 & $\begin{array}{l}\text { Reported } \\
\text { HFMD }\end{array}$ \\
\hline $\begin{array}{l}\text { Wang et al. } \\
\text { (2019) [27] }\end{array}$ & $\begin{array}{l}\text { Guangdong, } \\
\text { China }\end{array}$ & 2009-2012 & 911,640 & All & $\begin{array}{l}\text { Mean temperature; mean } \\
\text { relative humidity; mean } \\
\text { rainfall }\end{array}$ & $\begin{array}{l}\text { Bayesian spatiotemporal } \\
\text { model autocorrelation, } \\
\text { adjusting for long-term } \\
\text { time trend and } \\
\text { autocorrelation }\end{array}$ & None & Monthly & $\begin{array}{l}\text { Temperate } \\
\text { climate }\end{array}$ & $\mathrm{RR}$ & High & 7 & $\begin{array}{l}\text { Reported } \\
\text { HFMD }\end{array}$ \\
\hline $\begin{array}{l}\text { Zhu et al. } \\
\text { (2020) [42] }\end{array}$ & Wuxi, China & 2011-2017 & 107,906 & All & $\begin{array}{l}\text { Cumulative maximum } \\
\text { temperature; cumulative } \\
\text { minimum temperature }\end{array}$ & $\begin{array}{l}\text { DLNM, adjusting for } \\
\text { time-varying factors } \\
\text { and other } \\
\text { meteorological factors }\end{array}$ & $\begin{array}{l}0-16 \\
\text { days }\end{array}$ & Daily & $\begin{array}{l}\text { Temperate } \\
\text { climate }\end{array}$ & $\mathrm{RR}$ & High & 7 & $\begin{array}{l}\text { Reported } \\
\text { HFMD }\end{array}$ \\
\hline $\begin{array}{l}\text { Ji et al. } \\
\text { (2020) [43] }\end{array}$ & $\begin{array}{l}\text { Tianjin, } \\
\text { China }\end{array}$ & 2014-2018 & 70,027 & $\begin{array}{l}0-15 \\
\text { years }\end{array}$ & $\begin{array}{l}\text { Cumulative mean } \\
\text { temperature }\end{array}$ & $\begin{array}{l}\text { DLNM and a susceptible } \\
\text { infectious recovery } \\
\text { models, adjusting for } \\
\text { long-term trends, } \\
\text { seasonality, DOW }\end{array}$ & $\begin{array}{l}0-14 \\
\text { days }\end{array}$ & Daily & $\begin{array}{l}\text { Temperate } \\
\text { climate }\end{array}$ & $\mathrm{RR}$ & High & 8 & $\begin{array}{l}\text { Reported } \\
\text { HFMD }\end{array}$ \\
\hline $\begin{array}{l}\text { Guo et al. } \\
\text { (2020) [44] }\end{array}$ & Xi'an, China & 2009-2018 & 31,2018 & All & $\begin{array}{l}\text { Maximum temperature; } \\
\text { cumulative maximum } \\
\text { temperature }\end{array}$ & $\begin{array}{l}\text { DLNM, combined with } \\
\text { the GAM, adjusting for } \\
\text { long-term trends and } \\
\text { seasonality, and week }\end{array}$ & $\begin{array}{l}0-8 \\
\text { weeks }\end{array}$ & Weekly & $\begin{array}{l}\text { Temperate } \\
\text { climate }\end{array}$ & $\mathrm{RR}$ & High & 6 & $\begin{array}{l}\text { Reported } \\
\text { HFMD }\end{array}$ \\
\hline
\end{tabular}




\subsection{The Overall Effects}

The overall effects of ambient temperature and relative humidity at single-day lags are shown in Figures 2 and 3. The overall effects of other factors, including rainfall, wind speed, and sunshine duration, re shown in Figures S1-S3, respectively. The results suggested that ambient temperature (RR, 1.105; 95\% CI, 1.078-1.133), relative humidity (RR, 1.014; 95\% CI, 1.009-1.018), rainfall (RR, 1.001; 95\% CI, 1.000-1.001), and wind speed (RR, 1.036; 95\% CI, 1.013-1.059) had all been significantly associated with increased the risk of HFMD. The overall effect of sunshine duration (RR, $0.997 ; 95 \% \mathrm{CI}$, 0.975-1.018) on HFMD was not significant (Figure S3). The overall effects based on cumulative lag models also showed that ambient temperature (RR, 1.937; 95\% CI, 1.564-2.400) and relative humidity (RR, 1.154; 95\% CI, 1.030-1.294) were positively associated with the risk of HMFD (Figures S4 and S5). We observed not only significant associations of meteorological factors with HFMD risk, but also high heterogeneity (e.g., $\mathrm{I}^{2}$ is $99.6 \%$ for ambient temperature in Figure 2 and $95.7 \%$ for relative humidity in Figure 3$)$. Therefore, we decided to conduct subgroup analyses to explore the sources of heterogeneity.

\begin{tabular}{|c|c|c|c|}
\hline Study & & Relative Risk $(95 \% \mathrm{Cl})$ & $\%$ Weight \\
\hline Chen et al. (2014) [19] & 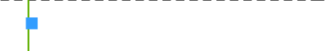 & $1.014(1.006-1.023)$ & 5.97 \\
\hline Zhu et al. (2016) [21] & $\longmapsto$ & $1.400(1.150-1.690)$ & 1.32 \\
\hline Yin et al. (2016) [18] & - & $1.050(1.040-1.060)$ & 5.95 \\
\hline Guo et al. (2016) [26] & - & $1.040(1.030-1.050)$ & 5.95 \\
\hline Hao et al. (2020) [13] & $\longmapsto$ & $1.945(1.570-2.408)$ & 1.12 \\
\hline Hao et al. (2020) [13] & $\longmapsto$ & $1.584(1.081-2.322)$ & 0.4 \\
\hline Xuan et al. (2017) [6] & F-1 & $1.056(1.003-1.109)$ & 4.84 \\
\hline Gou et al. (2018) [23] & - & $1.059(1.054-1.065)$ & 5.99 \\
\hline Gou et al. (2018) [23] & 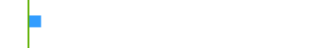 & $1.028(1.025-1.031)$ & 6 \\
\hline Gou et al. (2018) [23] & 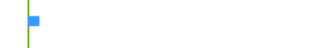 & $1.018(1.014-1.022)$ & 6 \\
\hline Onozuka et al. (2011) [24] & 1ー-1 & $1.120(1.032-1.198)$ & 3.92 \\
\hline Hii et al. (2011) [11] & - & $1.365(1.341-1.389)$ & 5.83 \\
\hline Hii et al. (2011) [11] & 昌 & $0.972(0.949-0.995)$ & 5.7 \\
\hline Tian et al. (2018) [40] & 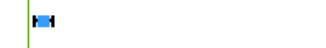 & $1.060(1.030-1.100)$ & 5.44 \\
\hline Kim et al. (2016) [25] & $=$ & $1.103(1.084-1.123)$ & 5.83 \\
\hline Xu et al. (2019) [10] & $\longmapsto$ & $2.474(2.065-2.965)$ & 1.46 \\
\hline Xu et al. (2019) [10] & $\longmapsto \longrightarrow$ & $1.494(1.253-1.781)$ & 1.52 \\
\hline Zhu et al. (2019) [8] & $\longmapsto$ & $1.100(0.940-1.290)$ & 1.77 \\
\hline Li et al. (2014) [7] & - & $1.105(1.104-1.106)$ & 6.01 \\
\hline Xuan et al. (2019) [14] & 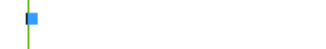 & $1.017(1.001-1.033)$ & 5.87 \\
\hline Wang et al. (2019) [27] & - & $1.045(1.021-1.069)$ & 5.72 \\
\hline Zhang et al. (2018) [38] & 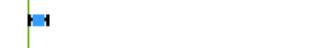 & $1.040(1.010-1.080)$ & 5.42 \\
\hline Guo et al. (2020) [44] & $\longmapsto$ & $1.560(1.350-1.810)$ & 1.97 \\
\hline Overall $\left(I^{2}=99.5, p=0.000\right)$ & + & $1.105(1.078-1.133)$ & 100 \\
\hline
\end{tabular}

Figure 2. Forest plot showing the effect of ambient temperature on the risk of HFMD based on single-day lag models $(n=23)$. 


\begin{tabular}{|c|c|c|c|}
\hline Study & & Relative Risk $(95 \% \mathrm{Cl})$ & $\%$ Weight \\
\hline Yang et al. (2018) [12] & $H$ & $1.080(1.040-1.130)$ & 1.07 \\
\hline Yang et al. (2018) [12] & $\mapsto$ & $1.050(1.020-1.080)$ & 1.96 \\
\hline Yang et al. (2018) [12] & $\longrightarrow$ & $0.920(0.780-1.080)$ & 0.08 \\
\hline Qi et al. (2018) [9] & 1 & $1.009(1.005-1.015)$ & 7.25 \\
\hline Chen et al. (2014) [19] & 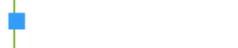 & $1.009(1.007-1.010)$ & 7.85 \\
\hline Bo et al. (2020) [22] & Her & $1.100(1.050-1.150)$ & 0.91 \\
\hline Bo et al. (2020) [22] & & $0.830(0.790-0.870)$ & 0.82 \\
\hline Guo et al. (2016) [26] & 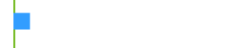 & $1.020(1.010-1.030)$ & 5.83 \\
\hline Hao et al. (2020) [13] & $\longmapsto$ & $1.553(1.322-1.824)$ & 0.08 \\
\hline Hao et al. (2020) [13] & $\longrightarrow$ & $0.805(0.597-1.086)$ & 0.02 \\
\hline Xuan et al. (2017) [6] & $\boldsymbol{P}$ & $1.017(1.008-1.026)$ & 6.13 \\
\hline Gou et al. (2018) [23] & 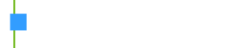 & $1.011(1.007-1.015)$ & 7.49 \\
\hline Gou et al. (2018) [23] & 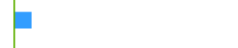 & $1.025(1.022-1.027)$ & 7.75 \\
\hline Gou et al. (2018) [23] & $\square$ & $1.047(1.024-1.072)$ & 2.68 \\
\hline Tian et al. (2018) [40] & 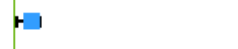 & $1.050(1.010-1.080)$ & 1.53 \\
\hline Kim et al. (2016) [25] & 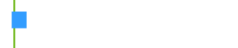 & $1.015(1.004-1.027)$ & 5.36 \\
\hline Yang et al. (2015) [41] & 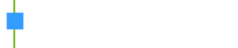 & $1.003(1.002-1.005)$ & 7.88 \\
\hline Zhu et al. (2019) [8] & $\longrightarrow$ & $1.320(1.020-1.710)$ & 0.03 \\
\hline Li et al. (2014) [7] & 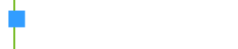 & $1.007(1.004-1.009)$ & 7.73 \\
\hline Wang et al. (2019) [27] & 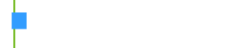 & $1.015(1.006-1.024)$ & 6.12 \\
\hline Xuan et al. (2019) [14] & 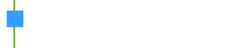 & $1.003(1.001-1.005)$ & 7.8 \\
\hline Xuan et al. (2019) [14] & 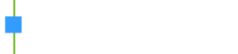 & $0.999(0.997-1.001)$ & 7.8 \\
\hline Zhang et al. (2018) [38] & 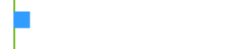 & $1.020(1.010-1.030)$ & 5.83 \\
\hline Overall $\left(I^{2}=95.7, p=0.000\right)$ & i & $1.014(1.009-1.018)$ & 100 \\
\hline
\end{tabular}

Figure 3. Forest plot showing the effect of relative humidity on the risk of HFMD based on single-day lag models $(n=23)$.

\subsection{Subgroup Analyses}

Our subgroup analysis focuses on the following variables to explore the sources of heterogeneity: exposure measures, exposure time resolution, regional climate, national income level, gender, and age. Tables 2 and 3 presented results based on the single-day lag models, and Table S2 showed results based on cumulative-day lag models (due to lack of data, this table only contains results on ambient temperature and relative humidity).

\subsubsection{Measure of Meteorological Factors}

In terms of ambient temperature, we found that for every $1{ }^{\circ} \mathrm{C}$ increase in average temperature, ( $n=16)$, the incidence of HFMD increased by $5.7 \%$ (RR, 1.057; 95\% CI, 1.030-1.084). Meanwhile, a $1{ }^{\circ} \mathrm{C}$ increase in maximum temperature $(n=4)$ was significantly associated with the incidence of HFMD (RR, 1.771; 95\% CI, 1.355-2.315). There was no significant association between the minimum temperature $(n=3)$ and the incidence of HFMD. For relative humidity, a $1 \%$ increase in average $(n=13)$ and maximum $(n=5)$ relative humidity can both increase the incidence of HFMD (RR, 1.017; 95\% CI, $1.011-1.024)$ and (RR, 1.015; 95\% CI, 1.005-1.026), respectively. The minimum relative humidity $(n=4)$ had no significant association with the incidence of HFMD. As for rainfall and wind speed, the included articles mainly used average values, and they were all positively associated with the risk of HFMD. 
Table 2. Subgroup analysis for the increased HFMD risk associated with ambient temperature and relative humidity based on single-day lag models.

\begin{tabular}{|c|c|c|c|c|c|c|}
\hline \multirow{2}{*}{ Subgroup Types } & \multicolumn{3}{|c|}{ Ambient Temperature } & \multicolumn{3}{|c|}{ Relative Humidity } \\
\hline & $n$ & $\mathrm{I}^{2} \%, p$-Value & Pooled RR $(95 \%$ CI) & $n$ & $\mathbf{I}^{2} \%, p$-Value & Pooled RR (95\% CI) \\
\hline \multicolumn{7}{|l|}{ Measure } \\
\hline Mean & 15 & $99.6 \%, p=0.000$ & $1.057(1.030-1.084)$ & 13 & $95.8 \%, p=0.000$ & 1.017 (1.011-1.024) \\
\hline Maximum & 4 & $94.5 \%, p=0.000$ & $1.771(1.355-2.315)$ & 5 & $94.7 \%, p=0.000$ & 1.015 (1.005-1.026) \\
\hline Minimum & 3 & $93.0 \%, p=0.000$ & $1.288(0.896-1.853)$ & 4 & $95.0 \%, p=0.000$ & $0.899(0.782-1.034)$ \\
\hline \multicolumn{7}{|l|}{ Time resolution } \\
\hline Daily & 8 & $92.8 \%, p=0.000$ & $1.074(1.038-1.111)$ & 15 & $93.7 \%, p=0.000$ & 1.009 (1.004-1.014) \\
\hline Weekly & 12 & $99.7 \%, p=0.000$ & $1.121(1.084-1.161)$ & 5 & $96.4 \%, p=0.000$ & $1.018(1.008-1.028)$ \\
\hline Monthly & 1 & & $1.045(1.021-1.069)$ & 1 & & $1.015(1.006-1.024)$ \\
\hline \multicolumn{7}{|l|}{ Regional climate } \\
\hline Tropical & 4 & $99.6 \%, p=0.000$ & $1.093(0.917-1.303)$ & 3 & $89.8 \%, p=0.000$ & $1.004(0.999-1.009)$ \\
\hline Temperate & 15 & $99.6 \%, p=0.000$ & $1.103(1.074-1.133)$ & 18 & $95.1 \%, p=0.000$ & $1.017(1.012-1.022)$ \\
\hline \multicolumn{7}{|l|}{ HDI * } \\
\hline High & 21 & $99.6 \%, p=0.000$ & $1.114(1.085-1.144)$ & 20 & $95.5 \%, p=0.000$ & $1.016(1.011-1.022)$ \\
\hline Low & 2 & $49.1 \%, p=0.161$ & $1.028(0.994-1.063)$ & 3 & $89.8 \%, p=0.000$ & $1.004(0.999-1.009)$ \\
\hline \multicolumn{7}{|l|}{ Gender } \\
\hline Male & 5 & $97.2 \%, p=0.000$ & $1.195(1.085-1.317)$ & 5 & $86.3 \%, p=0.007$ & $1.008(1.002-1.014)$ \\
\hline Female & 5 & $96.8 \%, p=0.000$ & $1.196(1.073-1.334)$ & 5 & $84.3 \%, p=0.007$ & $1.006(1.000-1.012)$ \\
\hline \multicolumn{7}{|l|}{ Age } \\
\hline $0-5$ year & 10 & $95.1 \% . p=0.000$ & $1.101(1.052-1.152)$ & 10 & $83.9 \%, p=0.000$ & $1.010(1.004-1.016)$ \\
\hline$>5$ year & 8 & $77.9 \%, p=0.000$ & $1.037(0.996-1.080)$ & 6 & $16.7 \%, p=0.306$ & $1.002(0.999-1.006)$ \\
\hline
\end{tabular}

${ }^{*}$ HDI: Human Development Index.

Table 3. Subgroup analysis for the increased HFMD risk associated with rainfall, wind speed, and sunshine duration based on single-day lag models.

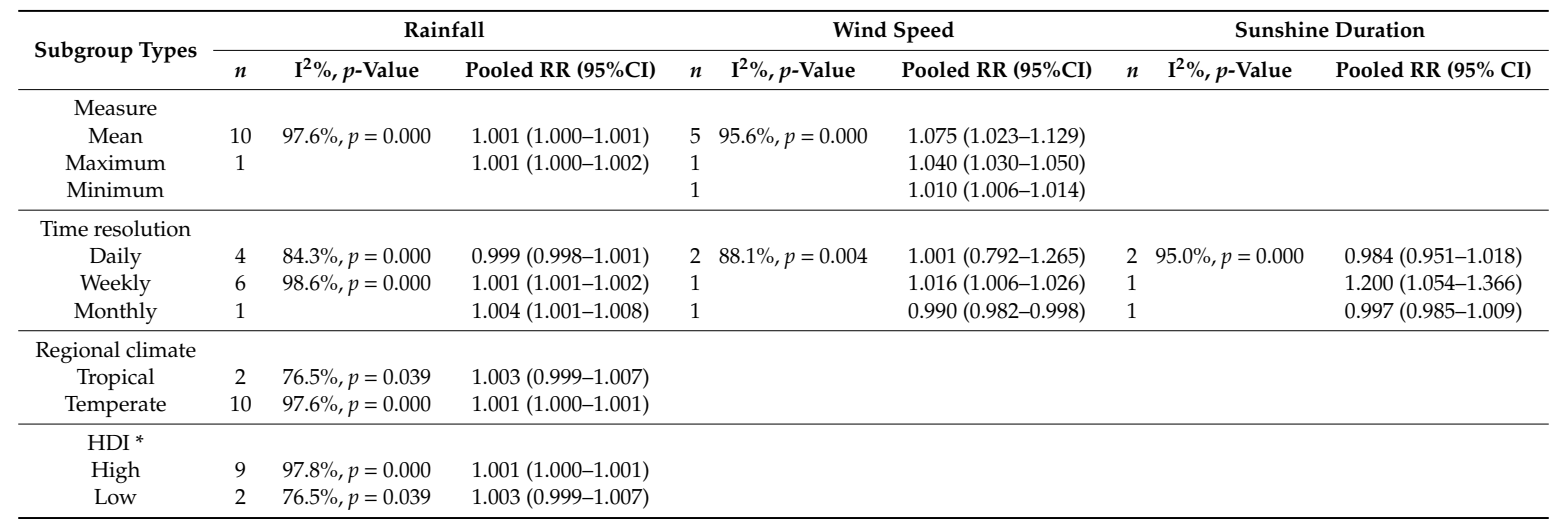

* HDI: Human Development Index.

\subsubsection{Exposure Time Resolution}

We observed that most of the included articles reported the RR values based on daily $(n=19)$ and weekly $(n=6)$ exposure values. Only one article used monthly exposure values to report results. Overall, for almost all meteorological factors, the RRs based on the daily and weekly exposure values are both significant $(p<0.05)$. Further, we found that the RRs generated by using the weekly exposure values are greater than the RRs produced by using the daily exposure values.

\subsubsection{Regional Climate}

The included articles were based on two climate types, namely temperate climate and tropical climate. There were more studies carried out in the temperate zone than in the tropics. The results based on single-day lag models showed that ambient temperature and relative humidity were significantly associated with HFMD risk in both temperate and tropical zones. A further observation revealed that the effects of ambient temperature, relative humidity, and rainfall on the risk of HFMD in the 
temperate zone are higher than that in the tropics. Meanwhile, the RR produced in the temperate zone under the cumulative lag model is also much higher than that in the tropical zone (Table S2).

\subsubsection{Human Development Index (HDI)}

Since there was no relevant data on national income, we used HDI as a proxy. Most of the included studies were conducted in areas with high HDI. We found that in the high HDI areas, ambient temperature, relative humidity, and rainfall were significantly associated with the risk of HFMD. Among them, the effect of ambient temperature and relative humidity on HFMD was higher in the high HDI areas than in the low HDI areas. The effect of rainfall in the high HDI areas was lower than those in the low HDI regions, but this effect was not statistically significant.

\subsubsection{Gender and Age}

Regarding ambient temperature and relative humidity, more than one third of the included articles reported the risks by gender and age groups. The effects of ambient temperature and relative humidity on HFMD were statistically significant in both genders, and there were no observable differences in the effect size of the two. For age groups, children under five are at higher risk than those over five.

\subsection{Sensitivity Analysis and Publication Bias}

We conducted two sensitivity analyses. In the first sensitivity analysis, we removed the extreme exposure values (maximum and minimum values) to verify whether the overall effect was affected by the extreme values. The results showed that the effects with extremes removed are comparable to those with extremes retained in the analysis, except for the ambient temperature, which had a higher effect on the risk of HFMD with extremes included than without them included (Table S3). Since a previous article has done a meta-analysis regarding meteorological factors and HFMD risk [15], our second sensitivity analysis aimed to compare the differences in the results reported in this article and results reflected in the updated literature. Comparing the two, we found that the overall effect of ambient temperature on HFMD risk has strengthened, while no obvious trend was observed for relative humidity over time (Table S4). We used both Begg's and Egger's tests to check whether publication bias exists. The results showed that articles regarding ambient temperature and relative humidity had some degrees of publication bias. The bias was not found on other meteorological factors (Table S5).

\section{Discussion}

This systematic review and meta-analysis explored the relationship between meteorological factors and the incidence of HFMD. We found that ambient temperature, relative humidity, rainfall, and wind speed were all significantly associated with the risk of HFMD. Further subgroup analyses also revealed that temperate regions, high-income countries, and children under five years of age are at high risk of developing HFMD. This article is the first study to include rainfall, wind speed, and sunshine duration into a meta-analysis of meteorological factors and HFMD, and it is also the first article to take the cumulative effect into the analysis.

We observed that both the maximum and mean ambient temperatures were significantly associated with the incidence of HFMD, and the effect of the maximum temperature was stronger than that of the mean temperature, which is consistent with the results of the previous meta-analysis [15]. Our sensitivity analysis also confirmed this result; that is, after removing the extreme high temperature from the analysis, the effect size is reduced (Table S3). Laboratory studies have found that high temperature can increase the infectivity of enteroviruses [45], and the virus multiplies faster in the high temperature environment as compared to that in the low temperature environment [46-48]. In hot weather, the public may increase outdoor activities, especially for children who are more likely to be infected through contact with recreational facilities and contaminated food [49,50]. Meanwhile, our results are consistent with most previous studies that the maximum and the mean relative humidity are positively associated with the incidence of HFMD. Moderate humidity may help the virus adhere 
to the surface of small objects, such as toys and food, which facilitates the survival and spread of the virus [51]. In addition, higher humidity affects the metabolism of children, limiting their sweat excretion and reducing their immunity [12].

Consistent with the result of [15], we found that the size of effect estimates generated by the use of weekly data are larger than the ones generated by the use of daily data. This may be due to the incubation period of the disease. HFMD has a short incubation period (typically three to seven days) and a long viral infection period. It can exist in infected people and people who have just recovered, which causes the second episode [52]. Therefore, there will always be a delay between when a patient notices the symptoms and this patient visits the doctor [53]. The delayed response to a clinical visit will affect the spread of the disease within a certain period of time [54]. Therefore, when measuring risk through the use of weekly data, it is easier to capture the association due to the time lags caused by the incubation period. For smaller geographic areas with low population density (such as rural areas), the use of weekly data can better investigate this association and improve statistical power [15].

Conventionally, HFMD is regarded as a tropical disease, though we found a higher meteorologically associated risk in temperate areas than in the tropics. This may be due to the various weather conditions in different climatic zones [26]. In temperate countries, the outbreak of HFMD usually occurs in summer or early fall [11]. Compared with tropical regions, temperate climates are more suitable for the survival and reproduction of enteroviruses. People living in temperate regions may be more involved in outdoor activities, thereby increasing their exposure to the virus. In contrast, although the commonly occurring rainy season in the tropics helps improve the breeding of the virus [55], once the rainfall exceeds a threshold, it will instead reduce people's outdoor activities, thereby reducing the spread of the virus [11]. This may explain that the highest incidence of HFMD was not in the tropics, but temperate areas. In addition, the frequency of extreme weather events occurring in the temperate zone was much lower than that in the tropics, which provides a good environment for the survival and reproduction of the virus.

Our study found that in high-income countries, ambient temperature, relative humidity, and rainfall all had a significant effect on the incidence of HFMD, while the previous meta-analysis did not find such statistical significance [15]. The possible reason is that the classification method of income level is different. Since there is no suitable standard, we used the 2019 United Nations Human Development Index as the basis to divide countries into high- and low-income countries. Our classification method has resulted in more areas defined as high-income countries, which increases the statistical power to detect the associations. Meanwhile, the high risk observed in high-income countries may also be due to people having better medical resources and stronger testing capabilities $[18,56-58]$. In high-income countries, almost all cases are recorded into the system, with few missed cases. This helps find the true associations with less selection bias. In the subsequent sensitivity analysis, we tried to classify income levels similar to what the previous meta-analysis had applied and divided countries into high- and middle-income countries and observed comparable results for the high-income countries (Table S4).

For the subgroup analysis on gender and age, we found that the risk of HFMD did not differ between genders. Children under five are at higher risk than those older than five. This may be due to their imperfect immune system development $[59,60]$. It has been reported that children under five years old lack antibodies such as Cox A16 and EV 71 to HFMD [61,62]. In addition, children sharing toys between playmates can also promote the spread of the virus $[18,63]$.

We conducted two sensitivity analyses. The first sensitivity analysis showed that the overall effect of including extreme temperatures is higher than that of excluding extreme temperatures (Table S3), which showed that it is important to prevent HFMD when extreme temperature events occur. The second sensitivity analysis showed that the association between meteorological factors and the risk of HFMD increased over time (Table S4). One possible reason is that global warming in recent years has led to higher ambient temperatures, which in turn has accelerated the spread of enteroviruses, thus increasing the risk of HFMD [64]. 
Our paper has the following limitations. First, we observed significant publication bias, especially on ambient temperature and relative humidity. The main reason for publication bias is that researchers tend to report statistically significant results and ignore those that are not significant. Some approaches to solve the problem of publication bias include: (1) trim and fill, (2) selection models, and (3) meta-regression. However, all of them have their own flaws. For example, the trim and fill method requires the elimination of articles that affect the symmetry of the funnel plot. The second method uses a selection model to screen the included articles, assuming that the probability of publication is associated with the $p$ value of its results, which ignores other factors that may affect publication. Meta-regression requires a large number of samples for the calculation to make sense [65]. Therefore, we chose to leave the publication bias as is in order to maintain the authenticity of the results. Relevant to the first limitation, our second limitation is that our results are highly heterogeneous, despite the fact that we used subgroup analysis to explore the source and sensitivity analysis to reduce the heterogeneity. Most of the included articles were carried out based on second-hand data, and they tended not to report insignificant results, even if such results did exist. As a result, we were not able to include these insignificant effects in our analysis. Third, we noticed that most of the included studies were conducted in the Asia-Pacific region, and there is currently a lack of relevant studies from other regions of the world. This may be another form of selection bias. Based on the above limitations, cautions need to be taken when our results are generalized to other regions of the world.

\section{Conclusions}

This systematic review and meta-analysis found that ambient temperature, relative humidity, rainfall, and wind speed were statistically significantly associated with HFMD. Extremely high temperature and relative humidity significantly increased the risk of HFMD. The overall impact of ambient temperature on the risk of HFMD has increased over time. Policy makers need to take preventive measures to deal with the impact of climate factors on the incidence of HFMD. Future research should focus on the non-Asia-Pacific region and relatively less studied meteorological factors, such as rainfall, wind speed, and sunshine duration.

Supplementary Materials: The following are available online at http://www.mdpi.com/1660-4601/17/21/8017/s1: Table S1: The quality of articles assessed by Newcastle Ottawa Scale (NOS), Table S2: Subgroup analysis for the increased HFMD risk associated with ambient temperature and relative humidity based on cumulative lag models, Table S3: The overall effect of each meteorological factor on the risk of HFMD (with or without extreme exposure values), Table S4: The differences in the effects of meteorological factors on HFMD between two meta-analyses (Chen et al. (2018) V.S the current study), Table S5: The $p$ values of Begg's and Egger's tests by meteorological factors based on single- and cumulative-day lag models, Tabl: Forest plot showing the effect of rainfall on the risk of HFMD based on single-day lag models $(n=11)$, Figure S2: Forest plot showing the effect of wind speed on the risk of HFMD based on single-day lag models $(n=7)$, Figure S3: Forest plot showing the effect of sunshine duration on the risk of HFMD based on single-day lag models $(n=5)$, Figure S4: Forest plot showing the effect of ambient temperature on the risk of HFMD based on cumulative-day lag models $(n=18)$, Figure S5: Forest plot showing the effect of relative humidity on the risk of HFMD based on cumulative-day lag models $(n=10)$.

Author Contributions: Conceptualization: Z.L. and Y.M.; methodology: Z.L.; software: Z.L.; validation: S.L. and Y.L.; formal analysis: Z.L.; investigation: Y.M.; resources: S.L. and H.X., data curation: Z.L; writing-original draft preparation: Z.L.; writing —review and editing: Z.L; visualization: Y.M.; supervision: S.L., H.X. and Y.L.; project administration: H.X. and Y.L.; funding acquisition: S.L. All authors have read and agreed to the published version of the manuscript.

Funding: This research received no external funding.

Conflicts of Interest: The authors declare no conflict of interest. 


\section{References}

1. World Health Organization (WHO). A Guide to Clinical Management and Public Health Response for Hand, Foot and Mouth Disease (HFMD). 2011. Available online: https:/www.who.int/whosis/whostat/2011/en/ (accessed on 8 May 2020).

2. Xing, W.; Liao, Q.; Viboud, C.; Zhang, J.; Sun, J.; Wu, J.T.; Chang, Z.; Liu, F.; Fang, V.J.; Zheng, Y.; et al. Hand, foot, and mouth disease in China, 2008-2012: An epidemiological study. Lancet Infect. Dis. 2014, 14, 308-318. [CrossRef]

3. Sabanathan, S.; Van Tan, L.; Thwaites, L.; Wills, B.; Qui, P.T.; Van Doorn, H.R. Enterovirus 71 related severe hand, foot and mouth disease outbreaks in South-East Asia: Current situation and ongoing challenges. J. Epidemiol. Community Health 2014, 68, 500-502. [CrossRef] [PubMed]

4. Ooi, M.H.; Wong, S.C.; Lewthwaite, P.; Cardosa, M.J.; Solomon, T. Clinical features, diagnosis, and management of enterovirus 71. Lancet Neurol. 2010, 9, 1097-1105. [CrossRef]

5. Wu, J.T.; Jit, M.; Zheng, Y.; Leung, K.; Xing, W.; Yang, J.; Liao, Q.; Cowling, B.J.; Yang, B.; Lau, E.H.; et al. Routine Pediatric Enterovirus 71 Vaccination in China: A Cost-Effectiveness Analysis. PLoS Med. 2016, 13, e1001975. [CrossRef]

6. Nguyen, H.X.; Chu, C.; Nguyen, H.L.T.; Nguyen, H.T.; Do, C.M.; Rutherford, S.; Phung, D. Temporal and spatial analysis of hand, foot, and mouth disease in relation to climate factors: A study in the Mekong Delta region, Vietnam. Sci. Total Environ. 2017, 581-582, 766-772. [CrossRef] [PubMed]

7. Li, T.; Yang, Z.; Di, B.; Wang, M. Hand-foot-and-mouth disease and weather factors in Guangzhou, southern China. Epidemiol. Infect. 2014, 142, 1741-1750. [CrossRef] [PubMed]

8. Zhu, H.S.; Chen, S.; Wang, M.Z.; Ou, J.M.; Xie, Z.H.; Huang, W.L.; Lin, J.W.; Ye, W.J. Analysis on association between incidence of hand foot and mouth disease and meteorological factors in Xiamen, 2013-2017. Chin. J. Epidemiol. 2019, 40, 531-536. [CrossRef]

9. Qi, H.; Chen, Y.; Xu, D.; Su, H.; Zhan, L.; Xu, Z.; Huang, Y.; He, Q.; Hu, Y.; Lynn, H.; et al. Impact of meteorological factors on the incidence of childhood hand, foot, and mouth disease (HFMD) analyzed by DLNMs-based time series approach. Infect. Dis. Poverty 2018, 7, 7. [CrossRef]

10. Xu, Z.; Hu, W.; Jiao, K.; Ren, C.; Jiang, B.; Ma, W. The effect of temperature on childhood hand, foot and mouth disease in Guangdong Province, China, 2010-2013: A multicity study. BMC Infect. Dis. 2019, $19,969$. [CrossRef]

11. Hii, Y.L.; Rocklöv, J.; Ng, N. Short term effects of weather on hand, foot and mouth disease. PLoS ONE 2011, 6, e16796. [CrossRef]

12. Yang, Y.; You, E.; Wu, J.; Zhang, W.; Jin, J.; Zhou, M.; Jiang, C.; Huang, F. Effects of relative humidity on childhood hand, foot, and mouth disease reinfection in Hefei, China. Sci. Total Environ. 2018, 630, 820-826. [CrossRef] [PubMed]

13. Hao, J.; Yang, Z.; Yang, W.; Huang, S.; Tian, L.; Zhu, Z.; Lu, Y.; Xiang, H.; Liu, S. Impact of Ambient Temperature and Relative Humidity on the Incidence of Hand-Foot-Mouth Disease in Wuhan, China. Int. J. Environ. Res. Public Health 2020, 17, 428. [CrossRef]

14. Nguyen, H.X.; Chu, C.; Tran, Q.D.; Rutherford, S.; Phung, D. Temporal relationships between climate variables and hand-foot-mouth disease: A multi-province study in the Mekong Delta Region, Vietnam. Int. J. Biometeorol. 2019, 64, 389-396. [CrossRef]

15. Cheng, Q.; Bai, L.; Zhang, Y.; Zhang, H.; Wang, S.; Xie, M.; Zhao, D.; Su, H. Ambient temperature, humidity and hand, foot, and mouth disease: A systematic review and meta-analysis. Sci. Total Environ. 2018, 625, 828-836. [CrossRef] [PubMed]

16. Shah, A.S.V.; Langrish, J.P.; Nair, H.; McAllister, D.A.; Hunter, A.L.; Donaldson, K.; Newby, D.E.; Mills, N.L. Global association of air pollution and heart failure: A systematic review and meta-analysis. Lancet 2013, 382, 1039-1048. [CrossRef]

17. Cai, Y.; Zhang, B.; Ke, W.; Feng, B.; Lin, H.; Xiao, J.; Zeng, W.; Li, X.; Tao, J.; Yang, Z.; et al. Associations of Short-Term and Long-Term Exposure to Ambient Air Pollutants With Hypertension: A Systematic Review and Meta-Analysis. Hypertension 2016, 68, 62-70. [CrossRef]

18. Yin, F.; Zhang, T.; Liu, L.; Lv, Q.; Li, X. The Association between Ambient Temperature and Childhood Hand, Foot, and Mouth Disease in Chengdu, China: A Distributed Lag Non-linear Analysis. Sci. Rep. 2016, 6, 27305. [CrossRef] 
19. Chen, C.; Lin, H.; Li, X.; Lang, L.; Xiao, X.; Ding, P.; He, P.; Zhang, Y.; Wang, M.; Liu, Q. Short-term effects of meteorological factors on children hand, foot and mouth disease in Guangzhou, China. Int. J. Biometeorol. 2014, 58, 1605-1614. [CrossRef]

20. Zhang, Q.; Zhou, M.; Yang, Y.; You, E.; Wu, J.; Zhang, W.; Jin, J.; Huang, F. Short-term effects of extreme meteorological factors on childhood hand, foot, and mouth disease reinfection in Hefei, China: A distributed lag non-linear analysis. Sci. Total Environ. 2019, 65, 839-848. [CrossRef]

21. Zhu, L.; Wang, X.; Guo, Y.; Xu, J.; Xue, F.; Liu, Y. Assessment of temperature effect on childhood hand, foot and mouth disease incidence (0-5 years) and associated effect modifiers: A 17 cities study in Shandong Province, China, 2007-2012. Sci. Total Environ. 2016, 551-552, 452-459. [CrossRef]

22. Bo, Z.; Ma, Y.; Chang, Z.; Zhang, T.; Liu, F.; Zhao, X.; Long, L.; Yi, X.; Xiao, X.; Li, Z. The spatial heterogeneity of the associations between relative humidity and pediatric hand, foot and mouth disease: Evidence from a nation-wide multicity study from mainland China. Sci. Total Environ. 2020, 707, 136103. [CrossRef] [PubMed]

23. Gou, F.; Liu, X.; He, J.; Liu, D.; Cheng, Y.; Liu, H.; Yang, X.; Wei, K.; Zheng, Y.; Jiang, X.; et al. Different responses of weather factors on hand, foot and mouth disease in three different climate areas of Gansu, China. BMC Infect. Dis. 2018, 18, 15. [CrossRef] [PubMed]

24. Onozuka, D.; Hashizume, M. The influence of temperature and humidity on the incidence of hand, foot, and mouth disease in Japan. Sci. Total Environ. 2011, 410-411, 119-125. [CrossRef] [PubMed]

25. Kim, B.I.; Ki, H.; Park, S.; Cho, E.; Chun, B.C. Effect of Climatic Factors on Hand, Foot, and Mouth Disease in South Korea, 2010-2013. PLoS ONE 2016, 11, e0157500. [CrossRef]

26. Guo, C.; Yang, J.; Guo, Y.; Ou, Q.Q.; Shen, S.Q.; Ou, C.Q.; Liu, Q.Y. Short-term effects of meteorological factors on pediatric hand, foot, and mouth disease in Guangdong, China: A multi-city time-series analysis. BMC Infect. Dis. 2016, 16, 524. [CrossRef] [PubMed]

27. Wang, Y.; Lai, Y.; Du, Z.; Zhang, W.; Feng, C.; Li, R.; Hao, Y. Spatiotemporal Distribution of Hand, Foot, and Mouth Disease in Guangdong Province, China and Potential Predictors, 2009-2012. Int. J. Environ. Res. Public Health 2019, 16, 1191. [CrossRef] [PubMed]

28. Yu, G.; Li, Y.; Cai, J.; Yu, D.; Tang, J.; Zhai, W.; Wei, Y.; Chen, S.; Chen, Q.; Qin, J. Short-term effects of meteorological factors and air pollution on childhood hand-foot-mouth disease in Guilin, China. Sci. Total Environ. 2019, 646, 460-470. [CrossRef]

29. Zhu, L.; Yuan, Z.; Wang, X.; Li, J.; Wang, L.; Liu, Y.; Xue, F.; Liu, Y. The Impact of Ambient Temperature on Childhood HFMD Incidence in Inland and Coastal Area: A Two-City Study in Shandong Province, China. Int. J. Environ. Res. Public Health 2015, 12, 8691-8704. [CrossRef]

30. Atkinson, R.W.; Cohen, A.; Mehta, S.; Anderson, H.R. Systematic review and meta-analysis of epidemiological time-series studies on outdoor air pollution and health in Asia. Air Qual. Atmos. Health 2012, 5, 383-391. [CrossRef]

31. Yang, B.Y.; Qian, Z.; Howard, S.W.; Vaughn, M.G.; Fan, S.J.; Liu, K.K.; Dong, G.H. Global association between ambient air pollution and blood pressure: A systematic review and meta-analysis. Environ Pollut. 2018, 235, 576-588. [CrossRef]

32. Biggerstaff, B.J.; Jackson, D. The exact distribution of Cochran's heterogeneity statistic in one-way random effects meta-analysis. Stat. Med. 2008, 27, 6093-6110. [CrossRef]

33. Kulinskaya, E.; Dollinger, M.B.; Bjørkestøl, K. On the moments of Cochran's Q statistic under the null hypothesis, with application to the meta-analysis of risk difference. Res. Synth. Methods 2011, 2, 254-270. [CrossRef] [PubMed]

34. United Nations. Human Development Report 2019. Available online: http://hdr.undp.org/en/2019-report (accessed on 2 March 2020).

35. Begg, C.B.; Berlin, J.A. Publication bias and dissemination of clinical research. J. Natl. Cancer Inst. 1989, 81, 107-115. [CrossRef]

36. Egger, M.; Davey Smith, G.; Schneider, M.; Minder, C. Bias in meta-analysis detected by a simple, graphical test. BMJ 1997, 315, 629-634. [CrossRef] [PubMed]

37. Xu, M.; Yu, W.; Tong, S.; Jia, L.; Liang, F.; Pan, X. Non-Linear Association between Exposure to Ambient Temperature and Children's Hand-Foot-and-Mouth Disease in Beijing, China. PLoS ONE 2015, 10, e0126171. [CrossRef] [PubMed] 
38. Zhang, X.; Xu, C.; Xiao, G. Space-time heterogeneity of hand, foot and mouth disease in children and its potential driving factors in Henan, China. BMC Infect Dis. 2018, 18, 638. [CrossRef]

39. Wang, P.; Goggins, W.B.; Chan, E.Y. Hand, Foot and Mouth Disease in Hong Kong: A Time-Series Analysis on Its Relationship with Weather. PLoS ONE 2016, 11, e0161006. [CrossRef] [PubMed]

40. Tian, L.; Liang, F.; Xu, M.; Jia, L.; Pan, X.; Clements, A.C.A. Spatio-temporal analysis of the relationship between meteorological factors and hand-foot-mouth disease in Beijing, China. BMC Infect Dis. 2018, 18, 158. [CrossRef]

41. Yang, H.; Wu, J.; Cheng, J.; Wang, X.; Wen, L.; Li, K.; Su, H. Is high relative humidity associated with childhood hand, foot, and mouth disease in rural and urban areas? Public Health 2017, 142, 201-207. [CrossRef]

42. Zhu, J.; Shi, P.; Zhou, W.; Chen, X.; Zhang, X.; Huang, C.; Zhang, Q.; Zhu, X.; Xu, Q.; Gao, Y.; et al. Assessment of Temperature-Hand, Foot, and Mouth Disease Association and Its Variability across Urban and Rural Populations in Wuxi, China: A Distributed Lag Nonlinear Analysis. Am. J. Trop. Med. Hyg. 2020, tpmd200329. [CrossRef]

43. Ji, X.-Y.; Huang, L.-Y.; Song, J.; Fei, C.-N.; Liu, J.; Liu, H. Short-term effects of meteorological factors, air pollution, and sunspot on childhood hand, foot, and mouth disease in Tianjin, China: A new time series regression, 2014-2018. Environ. Sci. Pollut. Res. 2020, 27, 37022-37035. [CrossRef] [PubMed]

44. Guo, T.; Liu, J.; Chen, J.; Bai, Y.; Long, Y.; Chen, B.; Song, S.; Shao, Z.; Liu, K. Seasonal Distribution and Meteorological Factors Associated with Hand, Foot, and Mouth Disease among Children in Xi'an, Northwestern China. Am. J. Trop. Med. Hyg. 2020, 102, 1253-1262. [CrossRef] [PubMed]

45. Yeager, J.G.; O’Brien, R.T. Enterovirus inactivation in soil. Appl. Environ. Microbiol. 1979, 38, 694-701. [CrossRef]

46. Loevinsohn, M. Climatic warming and increased malaria incidence in Rwanda. Lancet 1994, 343, 714-718. [CrossRef]

47. Rogers, D.J.; Randolph, S.E. The Global Spread of Malaria in a Future, Warmer World. Science 2000, 289, 1763-1766. [CrossRef]

48. Harvell, C.D.; Mitchell, C.E.; Ward, J.R.; Altizer, S.; Dobson, A.P.; Ostfeld, R.S.; Samuel, M.D. Climate Warming and Disease Risks for Terrestrial and Marine Biota. Science 2002, 296, 2158-2162. [CrossRef]

49. Xu, Z.; Sheffield, P.E.; Hu, W.; Su, H.; Yu, W.; Qi, X.; Tong, S. Climate Change and Children's Health-A Call for Research on What Works to Protect Children. Int. J. Environ. Res. Public Health 2012, 9, 3298-3316. [CrossRef]

50. Urashima, M.; Shindo, N.; Okabe, N. Seasonal models of herpangina and hand-foot-mouth disease to simulate annual fluctuations in urban warming in Tokyo. Jpn. J. Infect. Dis. 2003, 56, 48-53.

51. Gao, J.; Sun, Y.; Lu, Y.; Li, L. Impact of Ambient Humidity on Child Health: A Systematic Review. PLoS ONE 2014, 9, e112508. [CrossRef]

52. CDC. Hand, Foot, \& Mouth Disease (HFMD) Fast Facts. 2011. Available online: https://www.rightdiagnosis. com/artic/facts_about_hand_foot_mouth_disease_cdc_oc.htm (accessed on 9 July 2020).

53. Huang, Y.; Deng, T.; Yu, S.; Gu, J.; Huang, C.; Xiao, G.; Hao, Y. Effect of meteorological variables on the incidence of hand, foot, and mouth disease in children: A time-series analysis in Guangzhou, China. BMC Infect. Dis. 2013, 13, 134. [CrossRef]

54. Wang, Q.; Zhang, W.; Zhang, Y.; Yan, L.; Wang, S.; Zhang, J.; Sun, J.; Chang, Z.; Wang, Z. Clinical features of severe cases of hand, foot and mouth disease with EV71 virus infection in China. Arch. Med Sci. 2014, 10, 510-516. [CrossRef]

55. World Health Organization (WHO). Trends and Statistics. Communicable Disease Surveillance and Response. 2010. Available online: https://iris.wpro.who.int/handle/10665.1/10647 (accessed on 20 March 2020).

56. Yuan, G.P.; Yang, Z.T. Factors affecting the incidence of HFMD case-control study. Zhongguo Gong Gong Wei Sheng Za Zhi 2011, 27, 1407-1409.

57. Gupta, M.; Wahl, B.; Adhikari, B.; Bar-Zeev, N.; Bhandari, S.; Coria, A.; Erchick, D.J.; Gupta, N.; Hariyani, S.; Kagucia, E.W.; et al. The need for COVID-19 research in low- and middle-income countries. Glob. Health Res. Policy 2020, 5, 1-4. [CrossRef]

58. James, N.; Lawson, K.; Acharya, Y. Evidence on result-based financing in maternal and child health in low- and middle-income countries: A systematic review. Glob. Health Res. Policy 2020, 5, 1-15. [CrossRef] [PubMed] 
59. Bouman, A.; Heineman, M.J.; Faas, M.M. Sex hormones and the immune response in humans. Hum. Reprod. Updat. 2005, 11, 411-423. [CrossRef] [PubMed]

60. Goksugur, N.; Goksugur, S. Hand, Foot, and Mouth Disease. N. Engl. J. Med. 2010, 362, e49. [CrossRef]

61. Zhu, Z.; Zhu, S.; Guo, X.; Wang, J.; Wang, D.; Yan, D.; Tan, X.; Tang, L.; Zhu, H.; Yang, Z.; et al. Retrospective seroepidemiology indicated that human enterovirus 71 and coxsackievirus A16 circulated wildly in central and southern China before large-scale outbreaks from 2008. Virol. J. 2010, 7, 300. [CrossRef]

62. Ji, Z.; Wang, X.; Zhang, C.; Miura, T.; Sano, D.; Funamizu, N.; Okabe, S. Occurrence of Hand-Foot-and-Mouth Disease Pathogens in Domestic Sewage and Secondary Effluent in Xi'an, China. Microbes Environ. 2012, 27, 288-292. [CrossRef]

63. Chang, L.-Y.; King, C.-C.; Hsu, K.-H.; Ning, H.-C.; Tsao, K.-C.; Li, C.-C.; Huang, Y.-C.; Shih, S.-R.; Chiou, S.-T.; Chen, P.-Y.; et al. Risk Factors of Enterovirus 71 Infection and Associated Hand, Foot, and Mouth Disease/Herpangina in Children During an Epidemic in Taiwan. Pediatrics 2002, 109, e88. [CrossRef] [PubMed]

64. Patz, J.A.; Campbell-Lendrum, D.; Holloway, T.; Foley, J.A. Impact of regional climate change on human health. Nat. Cell Biol. 2005, 438, 310-317. [CrossRef]

65. Bland, M. Meta-Analysis: Heterogenity and Publication Bias. 2006. Available online: http://martinbland.co. $\mathrm{uk} / \mathrm{msc} /$ (accessed on 20 March 2020).

Publisher's Note: MDPI stays neutral with regard to jurisdictional claims in published maps and institutional affiliations. 